\title{
Mass Nouns in a Logic of Classes as Many
}

\author{
Nino B. Cocchiarella \\ Indiana University
}

\begin{abstract}
A semantic analysis of mass nouns is given in terms of a logic of classes as many. In previous work it was shown that plural reference and predication for count nouns can be interpreted within this logic of classes as many in terms of the subclasses of the classes that are the extensions of those count nouns. A brief review of that account of plurals is given here and it is then shown how the same kind of interpretation can also be given for mass nouns.
\end{abstract}

Why is the semantics of mass nouns so problematic? One reason, apparently, is that unlike count nouns, which have determinate extensions, mass nouns have extensions that in some cases are said to be indeterminate. The objects in the extension of a count noun are unproblematic because those objects are generally discrete and well-delineated, and hence can be individuated. The objects in the extension of a mass noun, especially mass nouns for different kinds of stuff, are said to be "diffuse" and indeterminate, on the other hand, because there are often an indefinite number of ways to refer them separately as well as together as wholes. ${ }^{1}$

A number of proposals have been made and criticized about the extensions of mass nouns. ${ }^{2}$ We will not review those proposals and criticisms here but instead will present a proposal of our own based on what has been called "the simplest plan" of all. ${ }^{3}$ We will defend this "plan" in terms of a logical framework designed to represent a form of conceptualism in which the logical forms that represent the cognitive structure of our speech and mental acts are distinguished from the logical forms that represent the truth conditions and deductive relations of those acts. ${ }^{4}$

\section{The Simple Logic of Names}

Both count nouns and mass nouns are represented in our conceptualist framework as common names, the initial or primary role of which is to be adjoined

${ }^{1}$ Cp. Bunt 1981, p. 53.

${ }^{2}$ See Pelletier 1974 for a review and discussion of some of these proposals.

${ }^{3}$ Pelletier 74 , p. 94.

${ }^{4}$ We will restrict ourselves in this paper to a fragment of our more general conceptualist (or conceptual realist) framework. This fragment suffices to explain our proposal regarding the semantics of plural and mass noun reference and predication. For an account of the fuller framework see Cocchiarella 2007. 
to quantifiers, as in 'Every man', 'Some horse', 'All water' and 'Some gold'. We take the resulting quantifier phrases to stand for the referential concepts that are exercised in our speech and mental acts. The category of names in our logic also includes proper names, which when they are adjoined to the quantifiers $\exists$ and $\forall$ can be taken to represent the use of a proper name with and without existential presuppositions respectively. Our initial conceptualist logic of names consists of a free first-order logic of identity extended to include quantifiers over names, which we take to stand for "nominal" (or name) concepts. (Predicate constants will be added in the next section where our initial logic is extended to the logic of classes as many.)

The logic of names contains absolute as well as relative quantifier phrases, i.e., relative quantifier phrases such as $(\forall x A)$ and $(\exists x A)$, where $A$ is a name, common or proper, and complex or simple. Instead of $(\forall x$ Object $)$ and $(\exists y O b j e c t)$, however, we will use the standard quantifier forms $(\forall x)$ and $(\exists y)$ for the absolute quantifier phrases, which, as usual, are read as 'Every object' and 'Some object', respectively. We will use $x, y, z$, etc., with or without numerical subscripts, as object variables and $A, B, C$, with or without numerical subscripts, as name, or "nominal", variables. Complex names are formed by adjoining socalled "defining" or restrictive relative clauses to names. We will use ' $/$ ', as in $A / \varphi x$ to represent the adjunction of a formula $\varphi x$ to the name $A$ (which may itself be complex). We read $A / \varphi x$ as ' $A$ that is $\varphi x$ '. Thus, e.g., the quantifier phrase representing reference to a house that is white would be symbolized as $(\exists x$ House/White $(x))$.

Names and formulas are inductively defined simultaneously as follows: (1) every name variable (or constant) is a name; (2) for all object variables $x, y$, $(x=y)$ is a formula; and if $\varphi, \psi$ are formulas, $B$ is a name (complex or simple), and $x$ and $C$ are an object and a name variable respectively, then (3) $\neg \varphi$, (4) $(\varphi \rightarrow \psi),(5)(\forall x) \varphi,(6)(\forall x B) \varphi$, and $(7)(\forall C) \varphi$ are formulas, and $(8) B / \varphi$ and (9) $/ \varphi$ are names, where $B / \varphi$ is read as ' $B$ that is $\varphi$ ' and $/ \varphi$ is read as 'object that is $\varphi$ ' (where the common name 'object' is dropped the way it with absolute quantifiers). The existential quantifier and other sentential connectives are understood as defined in the usual way. We assume the usual definitions of bondage and freedom for object variables and of the proper substitution of one object variable for another in a formula; and we assume as well the definitions of bondage and freedom of occurrences of name variables in formulas, and the proper substitution in a formula $\varphi$ of a name variable (or constant) $B$ for free occurrences of a name variable $C .^{5}$

The logical connections between relative and absolute quantifier phrases are given in the first two of the following meaning postulates. The other two postulates amount to export and import rules for quantifier phrases with complex names.

$$
\begin{gathered}
(\forall x A) \varphi \leftrightarrow(\forall x)[(\exists y A)(x=y) \rightarrow \varphi], \\
(\exists x A) \varphi \leftrightarrow(\exists x)[(\exists y A)(x=y) \wedge \varphi],
\end{gathered}
$$

\footnotetext{
${ }^{5}$ For details see Cocchiarella 2001.
} 


$$
\begin{gathered}
(\forall x B / \varphi) \psi \leftrightarrow(\forall x B)[\varphi \rightarrow \psi], \\
(\exists x B / \varphi) \psi \leftrightarrow(\exists x B)[\varphi \wedge \psi] .
\end{gathered}
$$

The axioms for the simple logic of names are described in the appendix where additional axioms for the logic of classes as many, which we will describe shortly, are also listed. The following are theorems that indicate some of the more interesting connections between the absolute and relative quantifiers of this logic:

$$
\begin{aligned}
& \vdash(\forall x) \varphi \rightarrow(\forall x A) \varphi . \\
& \vdash(\forall x A) \varphi \rightarrow[(\exists z A)(y=z) \rightarrow \varphi(y / x)], \quad \text { where } y \text { is free for } x \text { in } \varphi . \\
& \vdash(\exists x A)(y=x) \rightarrow(\exists x)(y=x) . \\
& \vdash(\forall x) \varphi \leftrightarrow(\forall A)(\forall x A) \varphi, \quad \text { where } A \text { is not free in } \varphi .
\end{aligned}
$$

Common names are not predicates, we want to emphasize; but there is an obvious connection, which perhaps explains why they are generally taken as such in textbooks on first-order predicate logic. For example, where $A$ is a name, proper or common, and complex or simple, we can associate a predicate, e.g., $F_{A}$ with $A$ as follows: $F_{A}(x) \leftrightarrow(\exists y A)(x=y)$; that is, $x$ is $F_{A}$ iff $x$ is an $A$. The copula reading ' $x$ is an $A$ ' in $(\exists y A)(x=y)$ is not odd even when $A$ is represents a mass noun, as we will see, because in that case much will depend on how the quantifier phrase $(\exists y A)$ is interpreted.

In any case, as we have shown elsewhere, this simple logic of names is equivalent to monadic second-order predicate logic. ${ }^{6}$ It is noteworthy that Stanislaw Leśniewski's logic of names, which he also called ontology, is reducible to our conceptualist simple logic of names. ${ }^{7}$

\section{Classes as Many as the Extensions of Names}

In addition to the referential use of common names as parts of quantifier phrases, there is also a "denotative" use, as when we speak of mankind, by which we mean the totality, or entire group, of humans taken collectively - but not in the sense of a set or class as an abstract object. ${ }^{8}$ Thus, we say that Socrates is a member of mankind, as well as that Socrates is a man. Also, instead of 'mankind', we can use the plural of 'man' and say that Socrates is one among men. The common name 'man', in other words, can be transformed into an "object term" that can occur as an argument of predicates the way that "singular terms," i.e., terms that purport to denote a single object, can. Such an "object term" in the common name case does not denote a single object, however, e.g., a set or a class as an abstract object, and therefore it is not itself a "singular term," which does not mean that it cannot be a value of the "object" variables. Nor does

\footnotetext{
${ }^{6}$ See, e.g., Cocchiarella 2001 or Cocchiarella 2008, Chapter 10.

${ }^{7}$ For proof of this claim see Cocchiarella 2001 or Cocchiarella 2008, Chapter 10.

${ }^{8}$ See, e.g., Sellars 1963 , p. 253 . We note here that there is also a "denotative" use of proper names as well, with or without existential presuppositions, which is the only use usually recognized by most logicians today.
} 
'mankind' denote the concept that 'man' stands for when occurring as part of a quantifier phrase. Rather, what 'mankind' denotes as an object term is the extension of the concept that the common noun 'man' stands for; only it is not an extension in the sense of a class as an abstract object, but in the sense of a plurality, or what Bertrand Russell once called a class as many.

The plurality of individuals that an expression such as 'mankind' denotes is a class as many, as opposed to a class as one. A class as many does not always have to be a plurality, i.e., many. It could consist of just a single object, as when a common name such as 'moon of the Earth' has just one object in its extension. But in that case the class as many is the same as that one object. On the other hand, there is no class as many that is empty. ${ }^{9}$

There are three important features of the notion of a class as many as the extension of a common name. The first is that a vacuous common name, i.e., a common name that names nothing, has no extension, which is not the same as having an empty class as its extension. Thus, according to Russell, "there is no such thing as the null class, though there are null class-concepts," i.e., commonname concepts that have no extension. ${ }^{10}$ The second feature, as already noted, is that the extension of a common name that names just one thing is just that one thing. And the third is that, unlike sets, classes as many as the extensions of names are literally made up of their members so that when they have more than one member they are merely pluralities (Vielheiten), or "plural objects," and not things that can themselves be members of classes. Thus, according to Russell, "though terms [i.e., objects] may be said to belong to ... [a] class, the class [as a plurality] must not be treated as itself a single logical subject." 11 It is this feature of not being a member of any class as many that represents the non-individuality of a class as many as a plurality, which, as a "plural object," can nevertheless be a value of the bound object variables of our logic. In other words, not all "objects" in our logic need be single objects, i.e., individuals in the traditional sense. This, of course, is an extended, nonstandard use of the word 'object', which we will continue to use for heuristic purposes. ${ }^{12}$

Once we allow names, including complex common names, to be transformed into object terms we need a variable-binding operator that generates complex names the way the $\lambda$-operator generates complex predicates. We will use the cap-notation with brackets, $[\hat{x} A / \ldots x \ldots]$, for this purpose. Accordingly, where $A$ is a name, proper or common, complex or simple, we take $[\hat{x} A]$ to be a complex name, but one in which the variable $x$ is bound. Thus, where $A$ is a name

\footnotetext{
${ }^{9}$ See Russell 1903, $\S \S 69-70$.

${ }^{10}$ It should be remembered that in free logic being a substituend of free object variablesi.e., being an "object term" - is not the same as denoting a value of the bound object variables. In free logic, in other words, some object terms may denote nothing. This is the case, e.g., with the object term corresponding a null concept, e.g., the name concept object-that-is-notself-identical, in symbols $/(x \neq x)$.

${ }^{11}$ Russell 1903, §70.

${ }^{12}$ This heuristic use of the word 'object' is analogous to Frege's explanation of concepts as unsaturated functions, and in particular when he speaks of the object expression 'the concept horse' as not denoting an object. Or as the Wittgenstein of the Tractatus put it: some things cannot be said but can only be shown (in our logic).
} 
and $\varphi$ is a formula, $[\hat{x} A],[\hat{x} A / \varphi]$, and $[\hat{x} / \varphi]$ are names in which all of the free occurrences of $x$ in $A$ and $\varphi$ are bound. We read these expressions as follows: $[\hat{x} A]$ is read as 'the things (i.e., single objects) that are $A$ '; $[\hat{x} A / \varphi]$ is read as 'the things that are $A$ that are $\varphi$ '; and $[\hat{x} / \varphi]$ is read as 'the things that are $\varphi$ '. (It should be kept in mind here that only single objects, i.e., things or individuals, can be members of classes as many.)

Let us now extend the simultaneous inductive definition of names and formulas given in the previous section so as to include names of this complex form and $n$-place predicate constants (for $n \in \omega$ ) as well. ${ }^{13}$ Note that we now have formulas of the form $(\forall y[\hat{x} A]) \varphi(y / x)$, as well as those of the form $(\forall x A) \varphi x$ and $(\forall y A(y / x)) \varphi(y / x) .{ }^{14}$ The first of these forms is reducible to the last because of the addition of the following axiom schema to the axioms for the simple logic of names:

$$
(\forall y[\hat{x} A]) \varphi \leftrightarrow(\forall y A(y / x)) \varphi, \quad \text { where } y \text { does not occur in } A \text {. }
$$

Other additional axioms, such as that $A=[\hat{x} A]$, are given in the appendix.

Note that it is just as natural to speak of membership in a class as many (in the sense of being one among the many) as it is of membership in a set, or class as one; and with membership we can define inclusion, proper or otherwise, in the usual way.

Def: $x \in y \leftrightarrow(\exists A)[(y=A) \wedge(\exists z A)(x=z)]$.

Def: $x \subseteq y \leftrightarrow(\forall z)[z \in x \rightarrow z \in y]$.

Def: $x \subset y \leftrightarrow x \subseteq y \wedge y \nsubseteq x$.

Note that in the definition of $\in$ the occurrence of $A$ in $(y=A)$ is as an object term denoting the things that fall under the name concept that $A$ stands for, whereas the occurrence of $A$ in the quantifier phrase $(\exists z A)$ stands for the name concept itself.

Russell's paradox is not derivable in this logic, incidentally. The concept of being a class as many that is not a member of itself can be represented qua nominal concept, but it does not follow in free logic that its extension exists as a class as many. In other words, even though the Russell concept is a value of the (second-order) name variables, the extension of the concept is not a value of the (first-order) object variables. Thus, as the following definition indicates, the name, or nominal, concept of the Russell class can be defined in purely logical terms.

Def: $R u s=[\hat{x} /(\exists A)(x=A \wedge x \notin A)]$.

But instead of leading to a contradiction, the Russell class as many is easily shown not to exist (as a value of the object variables): that is, $\neg(\exists x)(x=R u s)$ is provable in this logic. Similarly, the empty class as many, namely, the class of things that are not self-identical, $[\hat{x} / x \neq x]$, also does not exist (as a value of

\footnotetext{
${ }^{13}$ See the appendix for the full definition.

${ }^{14}$ We take $A(y / x)$ and $\varphi(y / x)$ to be the result of properly substituting $y$ for $x$ in $A$ and $\varphi$, respectively. The slash ' $/$ ' in these expressions is not to be confused of course with the slash in the formation of a complex name $A / \varphi x$.
} 
the object variables); and neither does the universal class, $[\hat{x} /(x=x)]$, if there are at least two single objects, i.e., individuals, which we also call atoms. ${ }^{15}$ For convenience we use $\boldsymbol{\Lambda}$ for the empty class as many and $\mathbf{V}$ for the universal class as many.

The (complex) name for atoms is defined as follows:

Def: Atom $=[\hat{x} / \neg(\exists y)(y \subset x)]$.

Of course, by an atom we do not mean here either a physical or a phenomenological atom, but only an object that has a discrete and well-delineated individual nature, such as a physical object or event, regardless of the complexity otherwise of that object. This 'atom' terminology goes back to Nelson Goodman and the so-called Leonard-Goodman calculus of individuals, which when formulated in a free logic turns out to be reducible to our present logic of classes as many. ${ }^{16} \mathrm{We}$ retain the terminology also because Goodman's nominalistic dictum that things are identical if they have the same atoms is provable in our logic of classes as many. That is,

$$
(\forall x)(\forall y)[(\forall z \text { Atom })(z \in x \leftrightarrow z \in y) \rightarrow x=y]
$$

is a theorem of this logic, which is not itself committed to nominalism.

The following standard definitions of union, intersection and complement are relevant in noting how the overlap and part-whole relations of (atomistic) mereology are definable in our logic.

Def: $x \cup y=[\hat{z} / z \in x \vee z \in y]$.

Def: $x \cap y=[\hat{z} /(z \in x \wedge z \in y)]$.

Def: $\bar{x}=[\hat{z} / z \notin x]$.

The overlap relation can now be defined as follows:

Def: $x \circ y \leftrightarrow x \cap y \neq \Lambda$.

Identity in the calculus of individuals (as reconstructed in a free logic) is restricted to "existing" objects, i.e., values of the bound object variables, and so is the part-whole relation. To say that " $x$ exists" can be represented either by $(\exists z)(x=z)$ or simply by $x \circ x$. This restricted notion of identity is defined as follows.

Def. $x \approx y \leftrightarrow x=y \wedge x \circ x$.

And the part-whole relations are defined as restricted inclusion, proper or otherwise:

Def: $x \leq y \leftrightarrow x \circ x \wedge x \subseteq y$.

Def: $x<y \leftrightarrow x \leq y \wedge y \not \leq x$.

Given these definitions, the notion of an atom in the Leonard-Goodman Calculus of individuals is now no different than our present notion in the logic of classes as many. Finally, without going into the details here, it can easily be shown that all of the axioms and inference rules (and therefore theorems

\footnotetext{
${ }^{15}$ For details on these matters see Cocchiarella 2002 or Cocchiarella 2008, Chapter 11.

${ }^{16}$ See Leonard-Goodman 1940. See Eberle 1970, Chapter 2, for a reconstruction of the calculus of individuals in a free first-order logic.
} 
as well) of the Leonard-Goodman calculus of individuals (as reconstructed in Eberle 1970) are derivable in our logic of classes as many. In fact, all of the different calculi of individuals formulated in Eberle 1970, including those with as well as without Goodman's nominalistic (atomicity) thesis, can easily be shown to be reducible to the present logic of classes as many.

\section{Plural Reference and Predication}

Our analysis of plural reference and plural predication has two parts. The first deals with a logical analysis of plural reference and predication in our speech and mental acts. The second deals with the logical forms that represent the truth conditions of those acts in terms of our logic of classes as many. The logical forms representing our speech and mental acts are a part of the deductive machinery of our logic only insofar as they are connected by meaning postulates to the logical forms that represent their truth conditions.

We assume that we can distinguish in a given speech context the common names that represent count nouns from those that represent mass nouns. We do not assume, on the other hand, that there is an absolute, fixed distinction between count and mass nouns, but only that we can make such a distinction in particular contexts of use of language. ${ }^{17}$ Plural reference in English, of course, involves only count nouns.

We extend the simultaneous inductive definition of the meaningful (wellformed) expressions of our conceptualist framework to include the following clauses. These clauses are designed to represent plural reference and predication in our speech and mental acts.

1. if $A$ represents a common count noun (in a given context), then $A^{P}$ is a plural name (in that context);

2. if $A$ represents a common count noun (in a given context), $x$ is an object variable, and $\varphi x$ is a formula, then $[\hat{x} A / \varphi x]^{P}$ and $[\hat{x} / \varphi x]^{P}$ are plural names (in that context);

3. if $A / \varphi(x)$ represents a (complex) common count noun (in a given context), then $(A / \varphi x)^{P}$ is $A^{P} /[\lambda x \varphi x]^{P}(x)$ and $[\hat{x} A / \varphi x]^{P}$ is $\left[\hat{x} A^{P} /[\lambda x \varphi x]^{P}(x)\right]$;

4. if $F$ is a one-place predicate constant, or of the form $[\lambda x \varphi(x)]$, then $F^{P}$ is a one-place plural predicate constant; and

5. if $A^{P}$ is a plural name (in a given context), $x$ is an object variable, and $\varphi$ is a formula, then $\left(\forall x A^{P}\right) \varphi$ and $\left(\exists x A^{P}\right) \varphi$ are formulas.

In regard to clause (5), we read, e.g., ' $\left(\forall x \operatorname{Man}^{P}\right)$ ' as the plural phrase 'all men' and ' $\left(\exists x \operatorname{Man}^{P}\right)$ ' as the plural phrase 'some men', and similarly ' $\left.\forall x C a t^{P}\right)$ ' as 'all cats', and ' $\left(\exists x C a t^{P}\right)$ ' as 'some cats', etc. We note that a plural name is not a name simpliciter and that unlike the latter there is no rule for the "nominalization" of a plural name, i.e., its transformation into an object term. Note also that only monadic predicates are pluralized. A two-place relation $R$

\footnotetext{
${ }^{17}$ See, e.g., Pelletier 1975 , p. 456, regarding the notion of a "universal grinder" that can change a count noun into a mass noun in a given context.
} 
can be pluralized in either its first- or second-argument position, or even both, by using a $\lambda$-abstract, as, e.g.,

$$
\begin{gathered}
{[\lambda x R(x, y)]^{P},} \\
{[\lambda y R(x, y)]^{P},} \\
{\left[\lambda x\left[\lambda y[R(x, y)]^{P}(y)\right]^{P},\right.}
\end{gathered}
$$

respectively; and a similar observation applies to $n$-place predicates for $n>2 .{ }^{18}$

Our initial claim is that we can represent the truth conditions of sentences with plural quantifier phrases in a natural and intuitive way in terms of our logic of classes as many as pluralities. Consider, for example, The Geach-Kaplan sentence 'Some critics admire only each other'. Intuitively, the truth conditions of this sentence can be read as 'There is a group or collection of critics who admire only each other'. Now, if by a group or collection we mean only a class as many, i.e., a number or plurality of critics, then the logical form representing the truth conditions of this sentence would be as follows:

$$
(\exists x / x \subseteq[\hat{y} C r i t i c])(\forall y / y \in x)(\forall z)[\operatorname{Admire}(y, z) \rightarrow z \in x \wedge z \neq y] .
$$

The group or collection of critics being posited in this formula cannot be empty, because there is no empty class as many. But this formula does not exclude the possibility that the group of critics in question has only one member - and hence is identical with that one member - who in this case, let us assume, admires no one, and who therefore vacuously satisfies the condition:

$$
(\forall y / y \in x)(\forall z)[\operatorname{Admire}(y, z) \rightarrow z \in x \wedge z \neq y]
$$

Such a possibility does not seem to be part of the content of this sentence, i.e., one of the possible contexts in which it could be truthfully asserted, or so I have argued elsewhere; and for that reason I thought that the group or collection that a plural quantifier phrase referred to must consist of more than one object. ${ }^{19}$ I now think that such an interpretation is not correct and that even the situation in question, regardless of its oddity, should be allowed as a possible context of use of the sentence. The above analysis, in other words, does represent the correct truth conditions of the sentence.

This analysis is supported by the fact that the plural reference in 'All men are mortal', the speech act of which is represented as follows,

$$
\left(\forall x \operatorname{Man}^{P}\right) \operatorname{Mortal}^{P}(x),
$$

should be in terms of classes as many in general and not in terms of classes as many with two or more members. In other words, the truth conditions of this sentence should be represented as

$$
(\forall x / x \subseteq M a n) \operatorname{Mortal}^{P}(x) .
$$

\footnotetext{
${ }^{18}$ See Cocchiarella 2008, Chapter 11 for details. Adding $\lambda$-abstracts is a trivial extension of the logic of classes as many. We will not go into those details here, however.

${ }^{19}$ That in fact is how I represented the situation in Cocchiarella 2008 and 2006.
} 
In other words, it would be unnatural to restrict the plural reference of 'All men' to classes as many with two or more men. Thus, given that the plural 'some' should be dual to the plural 'all', then the appropriate meaning postulate for the plural 'Some' should be:

$$
\left(\exists x A^{P}\right) \varphi x \leftrightarrow(\exists x / x \subseteq A) \varphi x,
$$

and the meaning postulate for the plural 'All' should be:

$$
\left(\forall x A^{P}\right) \varphi x \leftrightarrow(\forall x / x \subseteq A) \varphi x .
$$

Note, incidentally, that the plural predicate phrase 'are mortal' is distributive over a plurality of which it is true. That is, where Mortal $^{P}$ is read as the plural predicate phrase 'are mortal', we have the following as a meaning postulate for $\operatorname{Mortal}^{P}$ :

$$
\operatorname{Mortal}^{P}(x) \leftrightarrow(\forall y / y \in x) \operatorname{Mortal}(y) .
$$

Hence, it follows that

$$
\left(\forall x \operatorname{Man}^{P}\right) \operatorname{Mortal}^{P}(x) \leftrightarrow(\forall x / x \subseteq \operatorname{Man})(\forall y / y \in x) \operatorname{Mortal}(y),
$$

is valid, and therefore because every single object is itself a class as many (consisting only of itself), it follows that

$$
(\forall x / x \subseteq \operatorname{Man})(\forall y / y \in x) \operatorname{Mortal}(y) \leftrightarrow(\forall x M a n) \operatorname{Mortal}(x)
$$

is valid as well. In other words, the truth conditions of the sentence 'All men are mortal' turn out to be exactly the way they are standardly understood in logic text books.

Of course not all plural predications will be reducible to singular predication the way that Mortal $^{P}$ is. Some examples of irreducible plural predications, which we will not go into here, can be found Cocchiarella 2005 and 2007.

Finally, we note that the logical form representing the cognitive structure of the speech act in which the Geach-Kaplan sentence 'Some critics admire only each other' is asserted can now be represented by

$$
\left(\exists x \operatorname{Critic}^{P}\right)[\lambda x(\forall y / y \in x)(\forall z)(\operatorname{Admire}(y, z) \rightarrow z \in x \wedge z \neq y)](x),
$$

which, by (SmCount) and $\lambda$-conversion reduces to our above analysis of its truth conditions:

$$
(\exists x / x \subseteq[\hat{y} C r i t i c])(\forall y / y \in x)(\forall z)[\operatorname{Admire}(y, z) \rightarrow z \in x \wedge z \neq y] .
$$

\section{On the Semantics of Mass Nouns}

As with our analysis of plural reference and predication we distinguish two parts to our analysis of mass nouns. The first deals with the logical forms that 
represent reference and predication with respect to mass nouns in our speech and mental acts. The second deals with the logical forms that perspicuously represent the truth conditions of those acts in terms of our logic of classes as many. Again, as with the logic of plurals, the logical forms that represent our speech and mental acts are a part of the deductive machinery of our logic only insofar as they are connected by meaning postulates to the logical forms that represent their truth conditions.

We retain our assumption that we can distinguish the common names that represent mass nouns in a given speech context from those that represent count nouns. We extend the simultaneous inductive definition of the meaningful (wellformed) expressions of our conceptualist framework to include the following clauses.

1. if $A$ represents a mass noun (in a given context), then $A^{M}$ is a mass name (in that context);

2. if $A$ represents a mass noun (in a given context), $x$ is an object variable, and $\varphi x$ is a formula, then $[\hat{x} A / \varphi x]^{M}$ and $[\hat{x} / \varphi x]^{M}$ are (complex) mass names (in that context);

3 . if $A / \varphi(x)$ represents a (complex) mass noun (in a given context), then $(A / \varphi x)^{M}$ is $A^{M} /[\lambda x \varphi x]^{M}(x)$ and $[\hat{x} A / \varphi x]^{M}$ is $\left[\hat{x} A^{M} /[\lambda x \varphi x]^{M}(x)\right]$; and

4. if $A^{M}$ is a mass name (in a given context), $x$ is an object variable, and $\varphi$ is a formula, then $\left(\forall x A^{M}\right) \varphi$ and $\left(\exists x A^{M}\right) \varphi$ are formulas.

We note that clause 3 is needed to allow for the representation of complex mass nouns, such as the mass noun 'polluted water', i.e., 'water that is polluted'. We turn now to our proposal regarding the extension of a mass noun.

The extensions of mass nouns are said to be indeterminate in some cases, we have said, because the objects that the mass nouns are true of in those cases are not always well-delineated the way the objects are in the extensions of count nouns. Generally we speak of those objects as parts of what the mass noun denotes, where the parts might themselves have subparts and overlap with other parts.

In referring to all, some, a lot of, etc., water, for example, we are referring to parts of water that have indefinitely many subparts that are again parts of water and that stand in the same relation to the larger part as the larger part to the whole. ${ }^{20}$ The various parts of water are thus not discrete, well-delineated "individuals" in the way that the minimal parts, i.e., the molecules, of water are discrete and well-delineated. ${ }^{21}$ In general, in other words, although the minimal parts of what a mass noun denotes are individuals, and hence can be individuated, it is the non-minimal parts that are problematic.

So why not assume that the extension of a mass nouns consists just of the minimal parts that it is true of? That proposal is what Pelletier calls "the simplest plan". ${ }^{22}$ The mass noun 'furniture', for example, would then denote

\footnotetext{
${ }^{20}$ Having indefinitely many subparts is not the same as having infinitely many subparts; that is, it does not mean that there is an infinite descent of subparts.

${ }^{21}$ Cp. Bunt 1981, p. 56.

${ }^{22}$ Pelletier 1974, p. 94.
} 
the class as many of individual pieces of furniture, i.e., the complete plurality of individual chairs, tables, etc., and 'jewelry' would denote the class as many (plurality) of individual pieces of jewelry, e.g., earrings, necklaces, etc. Mass nouns for elementary substances and chemical compounds would then denote the class as many, or plurality, of individual atoms and/or molecules of those substances and compounds.

The mass nouns that traditionally have been assumed not to have minimal parts are 'space' and 'time'. It is significant therefore that in fact space and time are "quantized" in modern quantum physics, and therefore do have minimal parts. The "Planck length" of $10^{-33} \mathrm{~cm}$. is the smallest length physically possible in quantum mechanics, and there is a smallest physically possible time as well, namely, the time it takes for light to cross the Planck length, which is $10^{-43}$ seconds. This means that space and time are not infinitely divisible after all. Determining the minimal parts of mass nouns such as 'wine', 'oil', 'leisure', 'history', etc., will be problematic, we agree, but in any case they do not have infinitely many subparts. In any case, we will assume, even if only for the sake of simplicity, that all mass nouns have extensions with determinate minimal parts that in principle can be individuated. This is not an a priori claim, it should be noted, but an assumption about our physical world. ${ }^{23}$

Pelletier claims that "this [simplest] proposal cannot work" because "it implies that for every mass term there will be some count term with the same denotation." ${ }^{24}$ Well, yes, for each mass name $A$ (as distinguished as such in a given context), there is the corresponding complex count name 'minimal part of $A^{\prime}$, which has a readily formulable counterpart in our logic. Certainly, the proposal does not imply, nor is it committed to, the claim that corresponding to each mass noun of English there is a simple count of English that has the same denotation. Pelletier nevertheless insists that the proposal is "obviously false" for three reasons, which we will take up in what follows.

Pelletier's first reason is that is the proposal "invokes an empirical claim about the meanings of mass terms (that there always is such a count noun)". Here the doubt about there always being a count noun can only be about there already being a corresponding simple count noun in English, which has nothing to do with our proposal. Also, the proposal says nothing at all about the "meanings" of mass nouns, nor even about the concepts they stand for as cognitive structures; rather, the proposal is only about the extensions of mass nouns. ${ }^{25}$

Pelletier's second objection is that

the extensions of 'water' and 'water molecules' would still have to

\footnotetext{
${ }^{23}$ The referee for this paper brought up the question of whether or not our discourse would be incoherent if there were no minimal parts, i.e., if "empirical atomism" were false. The referee also suggested that it would not matter for the semantics of mass nouns which way the facts turn out to be. These are important questions that deserve careful and lengthy discussion that goes beyond the scope of this paper.

${ }^{24}$ Ibid.

${ }^{25}$ It is unclear why Pelletier does not apply this criticism to the other proposals he considers about the extensions of mass nouns, even though they are about such strange entities as "quantities" or structural properties, etc.
} 
be distinct since 'water' is true of this puddle but 'water molecule' is not. But this is impossible because they have the same denotation." 26

Here, the critical notion is that of a mass noun being true of something such as a puddle. On our proposal, as we will see, not all of the objects that 'water' is true of (as a mass noun) are objects that 'water molecule' is true of even if 'water' and 'water molecule' have the same extension. Indeed, in general, on our proposal, not all of the objects that a mass noun is true of - such as a puddle that 'water' is true of - are in the extension of that mass noun-e.g., the puddle is not in the extension of 'water', which on our proposal consists of just the minimal parts of water - just as not all of the objects that a plural count noun is true of are single objects, i.e., individuals, in the extension of that count noun. Nor, on our proposal, will being-a-minimal-part-of $A$ be true of all the objects that the mass noun $A^{M}$ is true of-just as being one (or a single object) will not be true of all the pluralities or classes as many that a plural count noun is true of. The justices of the Supreme Court are not one, for example, but nine, even though only single individuals are in the extension of the count noun 'justice of the Supreme Court'.

Before explaining this in more detail, let us first take up Pelletier's third objection, which is connected with his second in that it concerns the copula and what it means to say that something is water. In particular, the copula, according to Pelletier,

would have to be regarded as ambiguous: sentences like 'This molecule is water' would be translated as

$$
a \in W
$$

but to translate 'This puddle is water' we would have to graft something like the calculus of individuals onto our set-theoretic base. ${ }^{27}$

Of course, as already noted in a previous section, the calculus of individuals is contained in our logic of classes as many, which means that no grafting of this calculus would be necessary; and, moreover, our logic is a logic of pluralities and not a set theory. But then, Pelletier's translation of 'This molecule is water' is not how the sentence is translated in our logic anyway.

In explaining our answer let us note that there is a use of the word 'some' in English that can be used only with count nouns in the plural and with mass nouns as normally understood. This is an observation that Pelletier himself has made. $^{28}$ What is significant about this? Well, it is precisely this use of the word 'some' that was used in our interpretation of plural count nouns in the previous section; and it is this interpretation that we propose to apply to mass nouns here as well. In particular, the plural count noun phrase 'some $A^{P}$, , in symbols,

\footnotetext{
${ }^{26}$ Ibid.

${ }^{27}$ Ibid.

${ }^{28}$ Ibid., p. 95.
} 
( $\left.\exists x A^{P}\right)$, was interpreted as $(\exists x A / x \subseteq A)$. And it is this same interpretation, we claim, that applies to mass names. That is, where $A^{M}$ is taken as a mass name in a given context, then 'Some $A^{M}$ ', which can also be read informally as 'Some part of $A$ ', is to be be interpreted as follows:

$$
\left(\exists x A^{M}\right) \varphi x \leftrightarrow(\exists x / x \subseteq A) \varphi x,
$$

(SmMass)

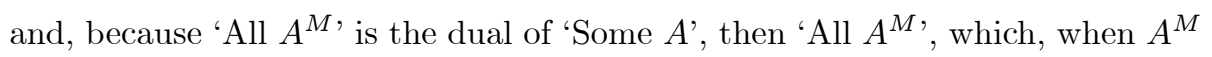
is a mass name, can also be read informally as 'Every part of $A$ ', is similarly interpreted as:

$$
\left(\forall x A^{M}\right) \varphi x \leftrightarrow(\forall x / x \subseteq A) \varphi x .
$$

In other words, the part-of $A$ relation, on our proposal that the extension of a mass name $A^{M}$ is the class as many of minimal parts of $A$, is just the inclusion relation in that extension - and of course there are more subclasses included in a class as many (of two or more objects) than there are things in that class. The objects that a mass noun is true of, in other words, are the different subclasses as many, i.e., the different pluralities, of the class of the minimal parts of $A$, including those minimal parts as well because each minimal part in our logic of classes as many is an individual and therefore identical with its singleton, which is also included in $A$. As individuals, moreover, the minimal parts are "single objects" whose individuation is unproblematic. Of course, the determination, even if not quite the "individuation", of the different parts of $A$ are on our interpretation no longer problematic as well. ${ }^{29}$

In regard to the copula, there is no difference, we maintain, between ' $a$ is a man' and ' $a$ is (some) water', where the parenthetical 'some' is the 'some' that is used with plural count names and mass names; and also, as Pelletier has noted, where this same parenthetical 'some' plays the role of an indefinite article for mass nouns. ${ }^{30}$ Thus, just as the analysis of ' $a$ is a man' is $(\exists x M a n)(a=x)$ in our logic, we also have the analysis of ' $a$ is (some) water' as $\left(\exists x\right.$ Water $\left.^{M}\right)(a=x)$. But note that although

$$
(\exists x \operatorname{Man})(a=x) \leftrightarrow a \in \operatorname{Man}
$$

is valid in the logic of classes as many (assuming that Man is a count name), the same equivalence does not apply to $\left(\exists x\right.$ Water $\left.^{M}\right)(a=x)$. Rather, given that $W_{a t e r}{ }^{M}$ is a mass name, what we have as valid by the postulate (SmMass) is:

$$
\left(\exists x \text { Water }^{M}\right)(a=x) \leftrightarrow(\exists x / x \subseteq \text { Water })(a=x),
$$

the right-hand side of which reduces simply to $a \subseteq$ Water. $^{31}$

\footnotetext{
${ }^{29}$ Not being individuals, the different pluralities or subclasses (of two or more members) of $A$ do not, of course, have an individuation in the sense that the members (minimal parts) of $A$ do.

${ }^{30}$ Ibid., p. 96.

${ }^{31}$ It is important to note that the common name Water occurs as a mass name only as part of the quantifier phrase in the left-hand side of the above biconditional, whereas it occurs as a common name simpliciter on the right-hand side, where on our proposal it is taken as denoting the minimal parts of water.
} 
The sentence 'This puddle is water' is now interpreted as $^{32}$

$$
\text { (This yPuddle })\left(\exists x \text { Water }^{M}(y=x)\right. \text {, }
$$

which, by (SmMass), reduces to

$$
\text { (This y Puddle) }(y \subseteq \text { Water }) \text {, }
$$

and not to

$$
\text { (This yPuddle) }(y \in \text { Water }) \text {. }
$$

Note that if Water $=[\hat{y} /$ Molecule-of-Water $(y)]$ is true, then this last formula is equivalent to the sentence 'This puddle is a molecule of water', which is false and not a consequence of our interpretation. In other words, even though 'water' is true of this puddle, 'water molecule' is not also true of this puddle. ${ }^{33}$ In other words, on our proposal, what 'water' is true of is not the same as what 'water molecule' is true of, even if they have the same extension - contrary to what Pelletier claims.

Note also that Pelletier's sentence 'This molecule is water', where 'water' is taken as a mass noun, now becomes

$$
\text { (This yMolecule) }\left(\exists x \text { Water }^{M}(y=x),\right.
$$

which reduces to

$$
\text { (This yMolecule })(y \subseteq \text { Water }),
$$

and not to

$$
\text { (This yMolecule })(y \in \text { Water }) \text {, }
$$

as Pelletier claimed. If in fact Water $=[\hat{y} /$ Molecule-of-Water $(y)]$ is true, then what the last formula represents is equivalent to the different sentence, 'This molecule is a molecule of water'. The sentence 'This molecule is water' is true because in the logic of classes as many each individual molecule $a$ of water is identical to its singleton, $[\hat{x} /(x=a)]$, and hence is part of Water, i.e., $a \subseteq$ Water is true as well as $a \in$ Water.

Pelletier gives another test as an adequacy condition that a proposal regarding the semantics of mass nouns must satisfy. This is that the following intuitively valid argument in English must come out valid in the logic being proposed:

This puddle is water.

Water is wet.

Therefore, this puddle is wet.

\footnotetext{
${ }^{32}$ In our conceptualist theory all referential phrases are represented as quantifier phrases. But one can just as well replace (This yPuddle) by an individual constant $a$ here. The expression This is of course taken here as a variable-binding operator.

${ }^{33}$ We are interpreting 'water molecule' here as the complex count noun 'thing that is a molecule of water', but this can be equally taken as the complex count noun 'molecule that is a minimal part of water'.
} 
Of course, 'water' is understood as a mass noun in this argument, and we understand the second premise as saying that all water is wet, which we can represent as $\left(\forall x W a t e r^{M}\right) W e t(x)$; and hence by the meaning postulate (AllMass) as $(\forall x / x \subseteq W$ ater $) W e t(x)$. The argument can therefore be reduced to the following clearly valid form in our logic of classes as many:

$$
\begin{gathered}
(\text { This yPuddle })(y \subseteq \text { Water }) . \\
(\forall x / x \subseteq \text { Water }) \text { Wet }(x) . \\
\therefore(\text { This yPuddle }) \text { Wet }(y) .
\end{gathered}
$$

Our interpretation of mass nouns within our logic of classes as many, we conclude, has an appropriate response to each of the objections put forth by Pelletier, and it validates the intuitively valid argument of English put forth as a test by Pelletier.

\section{Appendix}

In what follows we formally describe the logic of classes as many, which is the semantic basis for our conceptualist logic of plurals and mass nouns. ${ }^{34}$ By a formal language $L$ we understood a set of predicate and name constants instead of a set of predicates and object constants. There will be object constants in a formal language as well, but they will be generated from the name constants by a "nominalizing" transformation. (In our more comprehensive framework of conceptual realism, which we are not concerned with here, object constants are also generated from predicate constants by a nominalizing transformation.) We extend the simultaneous inductive definition of names and formulas given in section one for the simple logic of names to include names that are "nominalized" as follows:

If $L$ is a formal language, then:

(1) Every name variable or name constant in $L$ is a name of $L$;

(2) if $a, b, c_{0}, \ldots, c_{n-1}$ are either object variables, name variables or name constants in $L$, or names of $L$ of the form $[\hat{x} B]$, where $x$ is an object variable and $B$ is a name (complex or simple) of $L$, and $F$ is an $n$-place predicate constant in $L$, then $(a=b)$ and $F\left(c_{0}, \ldots, c_{n-1}\right)$ are formulas of $L$; and

if $\varphi, \psi$ are formulas of $L, B$ is a name (complex or simple) of $L$, and $x$ and $C$ are an object and a name variable, respectively, then

(3) $\neg \varphi$,

(4) $(\varphi \rightarrow \psi)$,

(5) $(\forall x) \varphi$

(6) $(\forall x B) \varphi$, and

(7) $(\forall C) \varphi$ are formulas of $L$, and

\footnotetext{
${ }^{34}$ For a set-theoretic semantics of the logic of classes as many see Cocchiarella 2002 or Cocchiarella 2007, Chapter 11, Appendix One. This set-theoretic semantics also establishes the consistency of the logic.
} 
(8) $B / \varphi$,

(9) $/ \varphi$, and

(10) $[\hat{x} B]$ are (complex) names of $L$.

The axioms of the simple logic of names are those of the free logic of identity plus the axioms for name quantifiers:

Axiom 1: All tautologous formulas (of any language $L$ being considered);

Axiom 2: $(\forall x)[\varphi \rightarrow \psi] \rightarrow[(\forall x) \varphi \rightarrow(\forall x) \psi]$;

Axiom 3: $(\forall C)[\varphi \rightarrow \psi] \rightarrow[(\forall C) \varphi \rightarrow(\forall C) \psi]$;

Axiom 4: $(\forall C) \varphi \rightarrow \varphi(B[x] / C)$, where $B$ is free for $C$ in $\varphi$ with respect to $x$;

Axiom 5: $\chi \rightarrow(\forall C) \chi, \quad$ where $C$ is not free in $\chi$;

Axiom 6: $\chi \rightarrow(\forall x) \chi, \quad$ where $x$ is not free in $\chi$;

Axiom 7: $(\forall x)(\exists y)(x=y), \quad$ where $x, y$ are different object variables;

Axiom 8: $x=x$;

Axiom 9: $x=y \rightarrow(\varphi \rightarrow \psi), \quad$ where $\varphi, \psi$ are atomic formulas and $\psi$ is obtained from $\varphi$ by replacing an occurrence of $y$ by $x^{35}$

Axiom 10: $(\forall x A) \varphi \leftrightarrow(\forall x)[(\exists y A)(x=y) \rightarrow \varphi], \quad$ where $x, y$ are different variables;

Axiom 11: $(\forall x A / \psi) \varphi \leftrightarrow(\forall x A)[\psi \rightarrow \varphi]$.

We assume as primitive inference rules modus ponens (MP) and universal generalization (UG) for absolute quantifiers indexed by either an object or a name variable. The rule of universal generalization for relative quantifiers,

$$
\text { if } \vdash \varphi \text {, then } \vdash(\forall x A) \varphi \text {, }
$$

is derivable by (UG) from Axiom 10. The usual laws for interchanging provably equivalent formulas and for rewriting bound variables are easily seen to be derivable as well. The universal instantiation law in free logic for object variables,

$$
(\exists x)(x=y) \rightarrow[(\forall x) \varphi \rightarrow \varphi(y / x)],
$$

where $x, y$ are distinct variables and $y$ is free for $x$ in $\varphi$, is derivable by Leibniz's law (LL), i.e., Axiom 9, (UG), Axioms 2 and 6, and tautologous transformations.

Once the logic of simple names is extended to include nominalized names, we then add the following axioms to the above:

Axiom 12: $(\forall y[\hat{x} A]) \varphi \leftrightarrow(\forall y A(y / x)) \varphi, \quad$ where $y$ does not occur in $A$. Axiom 13: $(\exists A)(A=[\hat{x} B]), \quad$ where $B$ is a name, complex or simple, and $A$ is a name variable that does not occur (free) in $B$.

\footnotetext{
${ }^{35}$ We note that the full version of Leibniz's law is derivable from this and the other axioms so long as no intensional operators are introduced into the logic.
} 
Axiom 14: $A=[\hat{x} A], \quad$ where $A$ is a simple name, i.e., a name variable or constant.

Axiom 15: $(\exists y)(y=[\hat{x} A]) \leftrightarrow(\exists x A)(x=x) \wedge(\forall x A)(\exists z$ Atom $)(x=z)$.

Axiom 16: $(\forall x A)(\forall y A)(x=y) \wedge(\forall x A)(\exists z$ Atom $)(x=z) \rightarrow$

$$
(\forall y A)(y=[\hat{x} A]) .
$$

Axiom 13 is a comprehension principle for complex names, and as such it is the analogue for complex names of the comprehension principle for complex predicates. What it says is that every complex name of the form $[\hat{x} B]$ is a value of the bound name variables, and therefore stands for a name concept. Axiom 14 tells us that the name concept $[\hat{x} A]$ is none other than the name concept $A$. Axiom 15 says that the extension of a name concept $A$ can be "object"-ified (as a value of the bound object variables) if, and only if, something is an $A$ and every $A$ is an atom. ${ }^{36}$ Axiom 16 concerns our second basic feature of classes as many; namely, that every atom, or individual, is identical with its singleton. In terms of a name concept $A$, the axiom stipulates that if at most one thing is an $A$ and that whatever is an $A$ is an atom, then whatever is an $A$ is identical to the extension of $A$, which in that case is a singleton if in fact anything is an $A$.

We note that the full unrestricted version of Leibniz's law is derivable from Axiom $9 .{ }^{37}$ If we extend this logic to include tense, modal or epistemic operators, then the full version of Leibniz's law will apply only to atoms, i.e., single objects or individuals. The restricted version for extensional contexts will still be applicable to pluralities, i.e., classes as many that have more than one member. Thus, adding such operators requires that we also add the following as an axiom schema of our general framework:

Axiom 17: $(\exists z$ Atom $)(x=z) \wedge(\exists z$ Atom $)(y=z) \rightarrow[x=y \rightarrow(\varphi \leftrightarrow \psi)]$,

where $\psi$ is obtained from $\varphi$ by replacing one or more free occurrences of $x$ by free occurrences of $y$.

This axiom is redundant, we want to emphasize, if we do not add any nonextensional contexts to the logic of classes as many.

Finally, given this understanding of identity and Leibniz's law, we add the axiom of extensionality to our logic of classes as many:

Axiom 18: $(\forall z)[z \in x \leftrightarrow z \in y] \rightarrow x=y$.

As already noted, Nelson Goodman's nominalistic dictum that things are identical if they have the same atoms is provable in our logic of classes as many regardless of how atoms are understood.

\footnotetext{
${ }^{36}$ That something is an $A$ is perspicuously symbolized by $(\exists y)(\exists x A)(y=x)$. But because $(\exists x A)(x=x) \leftrightarrow(\exists y)(\exists x A)(y=x)$ is provable, we use $(\exists x A)(x=x)$ as a shorter way of saying the same thing.

${ }^{37}$ Note that Axiom 9 is restricted to atomic formulas. An unrestricted version of LL for strictly extensional contexts is derivable by induction over the extensional formulas.
} 


\section{References}

[1] Bunt, Harry, 1981, "On the Why, the How, and the Whether of a Count/Mass Distinction among Adjectives," in Formal Methods in the Study of Language, Part 1, J.A.G. Groenendijk, T.M.V. Janssen, and M.J.B. Stokof, eds., Mathematish Centrum, Amsterdam.

[2] Cocchiarella, Nino B., 2001, "A Conceptualist Interpretation of Leśniewski's Ontology," History and Philosophy of Logic 22: 29-43.

[3] Cocchiarella, Nino B., 2002, "On the Logic of Classes as Many," Studia Logica Vol. 70, No. 3: 303-338.

[4] Cocchiarella, Nino B., 2005, "Denoting Concepts, Reference, and the Logic of Names, Classes as Many, Groups, and Plurals," in Linguistics and Philosophy, vol. 28 (2005): 135-179.

[5] Cocchiarella, Nino B., 2007, Formal Ontology and Conceptual Realism, Synthese Library vol, 339, Springer, Dordrecht.

[6] Eberle, Rolf A., 1970, Nominalistic Systems, Synthese Library, D. Reidel Pub. Co., Dordrecht.

[7] Leonard, H.S. and N. Goodman, 1940, "The Calculus of Individuals," The Journal of Symbolic Logic 5: 45-55.

[8] Pelletier, Francis J., 1974, "On Some Proposal for the Semantics of Mass Nouns," Journal of Philosophical Logic Vol. 3: 87-108.

[9] Pelletier, Francis J., 1975, "Non-Singular Reference: Some Preliminaries," Philosophia Vol. 5, No. 4: 451-465. (Reprinted in: Pelletier, Francis J., Mass Terms: Some Philosophical Problems, Reidel, Dordrecht, 1979, pp. $1-14)$.

[10] Russell, Bertrand, 1903, The Principles of Mathematics, second edition, Norton \& Co., N.Y., 1938.

[11] Sellars, Wilfrid F., 1960, "Grammar and Existence: A Preface to Ontology," Mind Vol. 69, No. 276: 499-533. (Reprinted in: Sellars Wilfrid F., Science, Perception and Reality, London, Routledge \& Kegan Paul, 1963 pp. 247-281). 